\title{
A Case Report on Acute Pancreatitis in a Patient With Coronavirus Disease 2019 (COVID-19) Pneumonia
}

\author{
Aviral Gupta ${ }^{1}$, Dharam P. Bansal ${ }^{1}$, Puneet Rijhwani ${ }^{1}$, Vipasha Singh ${ }^{2}$ \\ 1. Department of General Medicine, Mahatma Gandhi Medical College and Hospital, Jaipur, IND 2. Department of \\ Radiology, Mahatma Gandhi Medical College and Hospital, Jaipur, IND
}

Corresponding author: Aviral Gupta, aviral.aiims@gmail.com

\begin{abstract}
With the ongoing coronavirus disease 2019 (COVID-19) pandemic, there has been an explosion of scientific literature on the clinical manifestations and pathogenesis of severe acute respiratory syndrome coronavirus 2 (SARS-CoV-2) infection. Gastrointestinal symptoms occur in 15-20\% of COVID-19 patients; however, there have not been many case reports on acute pancreatitis in COVID-19 patients. The expression of ACE-2 ([angiotensin-converting enzyme 2] the host receptor for SARS-CoV-2) is very high in the pancreas, which might be a contributing factor, but the high expression is mainly localized to endocrine pancreas. This case report describes a case of a 25-year-old Indian female with COVID-19 with acute pancreatitis in the absence of any other known risk factors for pancreatitis.
\end{abstract}

Review began 04/20/2021 Review ended 04/21/2021 Published 04/22/2021

\section{(c) Copyright 2021}

Gupta et al. This is an open access article distributed under the terms of the Creative Commons Attribution License CC-BY 4.0., which permits unrestricted use, distribution, and reproduction in any medium, provided the original author and source are credited.
Categories: Internal Medicine, Gastroenterology, Infectious Disease

Keywords: coronavirus disease 2019, covid-19, sars-cov-2, acute pancreatitis, viral pancreatitis, amylase, lipase

\section{Introduction}

The identification of a novel coronavirus had led to a cluster of severe pneumonia cases in Wuhan, China, in December 2019. The disease was later designated as coronavirus disease 2019 (COVID-19) [1] by the World Health Organization on March 11, 2020 [2]. Since its inception, there have been more than 83.3 million cumulative cases and 1.8 million deaths worldwide as of January 5, 2021 [3]. The clinical spectrum of the disease ranges from asymptomatic infection [4] to critical life-threatening respiratory disease [5]. However, the organ involvement in COVID-19 is not limited to the respiratory system, and several other organ systems can be involved. Data analysis indicates the presence of six distinct clusters of symptoms of COVID19 [6], including gastrointestinal symptoms of varying severity. There has been evidence of pancreatic injury in COVID-19 patients, but this is often overlooked and the real prevalence of this complication is unknown. This case report highlights the case of a patient with COVID-19 pneumonia who developed acute pancreatitis.

\section{Case Presentation}

A 25-year-old Indian female with no significant past history presented to our emergency department with complaints of fever, headache, sore throat, dry cough, and loss of taste sensation for nine days, and abdominal pain with vomiting for two days. Her COVID reverse transcription polymerase chain reaction (RTPCR) test performed on the second day of her illness returned positive. Subsequent to her first positive RTPCR, the patient was started on oral favipiravir, tablet ivermectin $12 \mathrm{mg}$ once daily for three days, and other supportive therapy. The patient did not have shortness of breath at this time or a history of smoking or alcohol intake. On the eighth day of illness, the patient started experiencing severe, dull aching, epigastric pain radiating to the back, with vomiting. The investigations on her eighth day of illness revealed that her repeat COVID RT-PCR was positive, C-reactive protein was $18.62 \mathrm{mg} / \mathrm{L}$, D-dimer was highly elevated $(>5,000$ $\mathrm{ng} / \mathrm{mL}$ ) and serum lipase was 2,052.61 U/L (>3 times upper limit of normal). She was then admitted to our hospital on the ninth day of illness as a case of COVID-19 with suspected acute pancreatitis. On physical examination, her vitals were normal and epigastric tenderness was appreciated. Her investigations before and after admission are shown in Table 1 , indicating a pro-inflammatory and hyper-coagulable state of COVID-19.

\begin{tabular}{|c|c|c|c|c|c|c|}
\hline Parameter & Day 9 & Day 11 & Day 13 & Day 14 & Day 15 & Day 16 \\
\hline COVID RT-PCR & - & - & Negative & Negative & - & - \\
\hline TLC (cells $/ \mathrm{mm}^{3}$ ) & 9700 & 7010 & 6730 & 7400 & 5490 & 6400 \\
\hline Neutrophil (cells/mm³) & $7 / 00$ & 5570 & 5320 & 5700 & 4350 & 4780 \\
\hline
\end{tabular}




\section{Cureus}

\begin{tabular}{|c|c|c|c|c|c|c|}
\hline Lymphocyte (cells $/ \mathrm{mm}^{3}$ ) & 1300 & 940 & 1110 & 1200 & 870 & 1000 \\
\hline NLR & 5.7 & 5.92 & 4.79 & 4.9 & 5.0 & 4.78 \\
\hline Hemoglobin (g/dL) & 11.6 & 10.0 & 10.0 & 10.1 & 11.0 & 11.2 \\
\hline Platelet count $\left(x 10^{3} / \mathrm{mm}^{3}\right)$ & 190 & 184 & 183 & 236 & 267 & 256 \\
\hline Eosinophil (cells/mm³) & 100 & 80 & 0 & - & 0 & 90 \\
\hline CRP (mg/L) & 52.6 & 81.9 & 62.1 & 34.9 & 27.6 & 37.4 \\
\hline D-dimer (ng/mL) & 3627 & 11,100 & 1850 & 3600 & 442 & 606 \\
\hline NT-proBNP (pg/mL) & - & 3990 & 2320 & 5010 & - & - \\
\hline Troponin I & - & 0.021 & $<0.010$ & $<0.010$ & - & - \\
\hline Procalcitonin & 0.030 & 0.24 & 0.13 & 0.096 & - & - \\
\hline Serum amylase & 1814 & - & & & - & - \\
\hline Serum lipase & 11920 & - & 301 & 275.3 & 225 & 327 \\
\hline IL-6 & 514.6 & - & & & - & - \\
\hline Urea & 22.6 & 19.7 & 38 & 19.2 & - & - \\
\hline Creatinine & 0.8 & 0.7 & 0.5 & 0.6 & - & - \\
\hline Calcium & 9.2 & 8.7 & 9.0 & - & - & - \\
\hline Blood sugar & 83.8 & 81.1 & - & - & - & - \\
\hline ESR & 55 & - & - & - & - & - \\
\hline HbA1c & 5.83 & - & - & - & - & - \\
\hline SGOT & 24 & 23.4 & - & - & - & - \\
\hline SGPT & 25.4 & 18.7 & - & - & - & - \\
\hline ALP & 121.1 & 100.0 & - & - & - & - \\
\hline Serum bilirubin & 0.6 & 0.8 & - & - & - & - \\
\hline Ferritin & 44.9 & - & - & - & - & - \\
\hline Triglycerides & 97.4 & - & - & - & - & - \\
\hline Prothrombin time & - & 16.2 & - & - & - & - \\
\hline VDRL & Negative & & & & & \\
\hline Malaria antigen & Negative & & & & & \\
\hline HIV & Negative & & & & & \\
\hline HBsAg & Negative & & & & & \\
\hline Anti-HCV antibody & Negative & & & & & \\
\hline Blood culture & Negative & & & & & \\
\hline TSH & 2.54 & & & & & \\
\hline
\end{tabular}

\section{TABLE 1: Laboratory investigations of the patient on days of illness.}

ALP, alkaline phosphatase; COVID, coronavirus disease 2019; CRP, C-reactive protein; HIV, human immunodeficiency virus; ESR, erythrocyte sedimentation rate; HBsAg, hepatitis B surface antigen; HbA1c, hemoglobin A1c; HCV, hepatitis C virus; IL-6, interleukin-6; NLR, neutrophil-tolymphocyte ratio; NT-proBNP, N-terminal-pro-B-type natriuretic peptide; PCR, reverse transcription polymerase chain reaction; SGOT, serum glutamic oxaloacetic transaminase; SGPT, serum glutamic pyruvic transaminase; TLC, total leucocyte count; TSH, thyroid-stimulating hormone; VDRL, Venereal Disease Research Laboratory test 


\section{Cureus}

A contrast-enhanced CT of the whole abdomen showed a bulky pancreas with poor enhancement, no parenchymal necrosis or calcification (Figures 1,2), with soft tissue stranding and inflammatory changes in peripancreatic and mesenteric fat; renal and lateroconal fascial thickening (Figure 3); and mild free fluid in peritoneal cavity and pelvis (Figure 4); which further confirmed our diagnosis of acute interstitial pancreatitis (fulfilling all three criteria of acute pancreatitis as per the revised Atlanta classification) with mild ascites (modified CT Severity Index - 6).

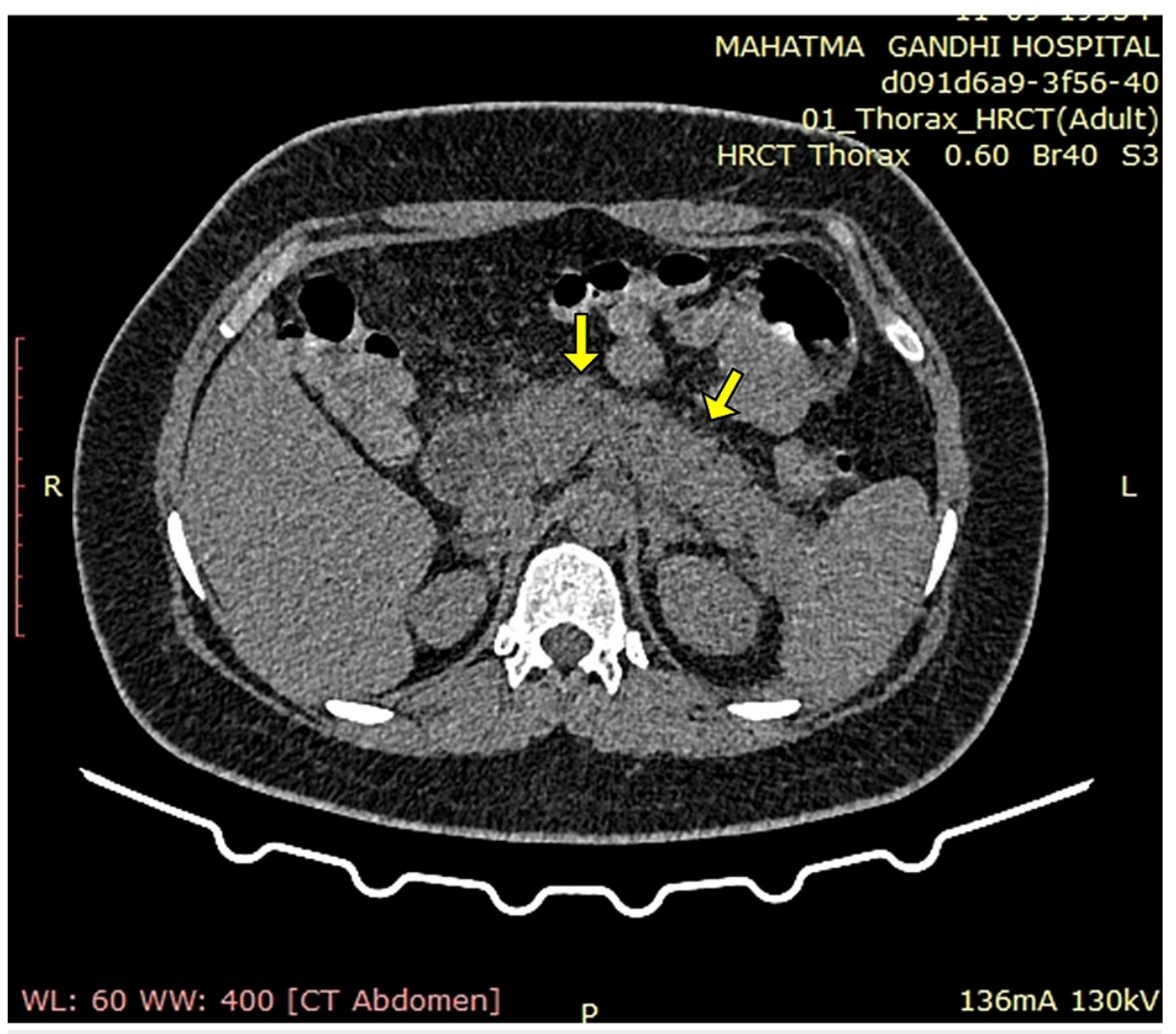

FIGURE 1: Non-contrast CT of the abdomen (axial section) showing diffusely bulky pancreas with no areas of calcification noted (solid yellow arrows). 


\section{Cureus}

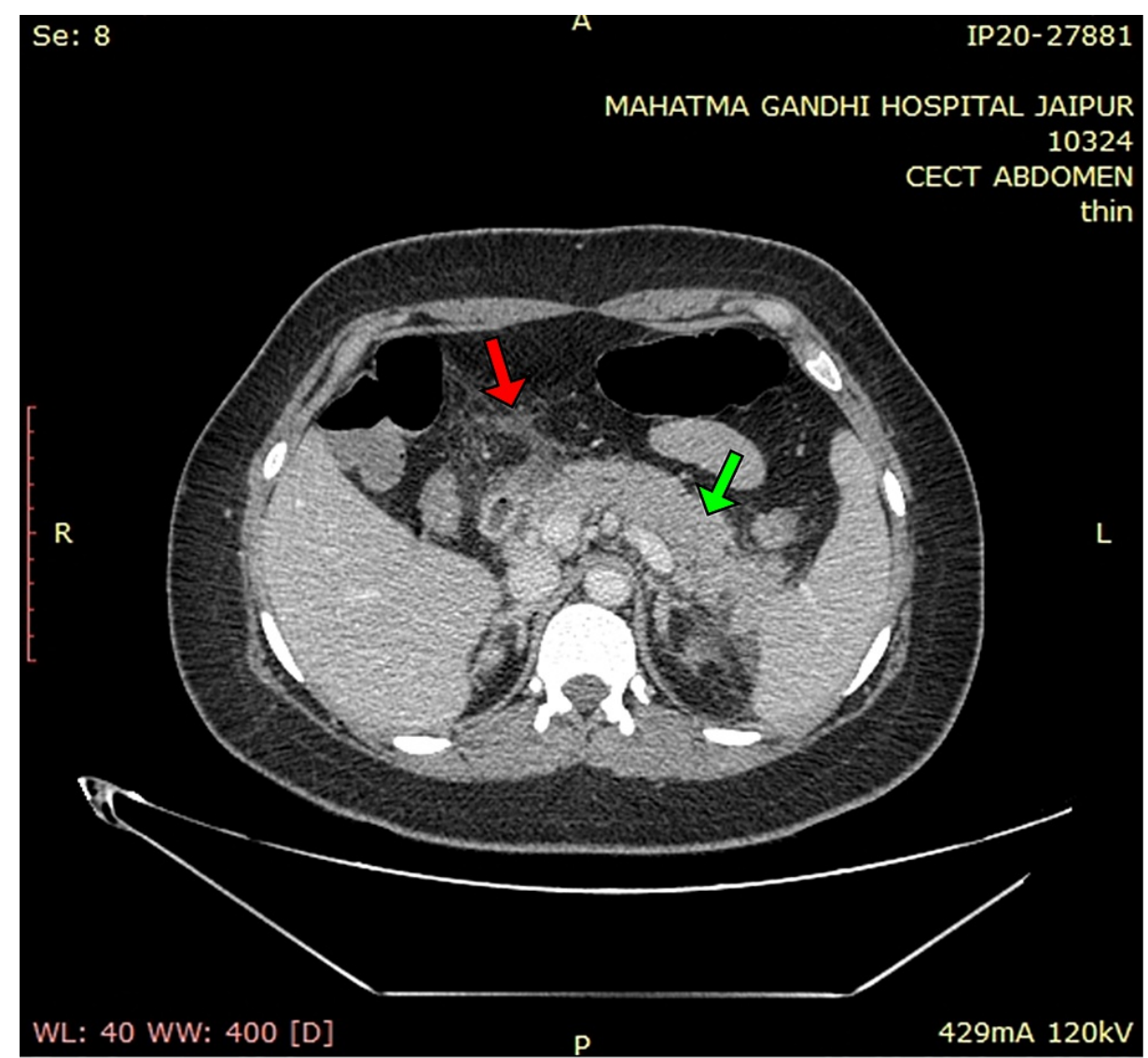

FIGURE 2: Contrast-enhanced CT of the abdomen (axial section) showing poorly enhancing, bulky pancreas, with no areas of necrosis (solid green arrow), pancreatic duct not dilated, and peri-pancreatic fat stranding (solid red arrow). 


\section{Cureus}

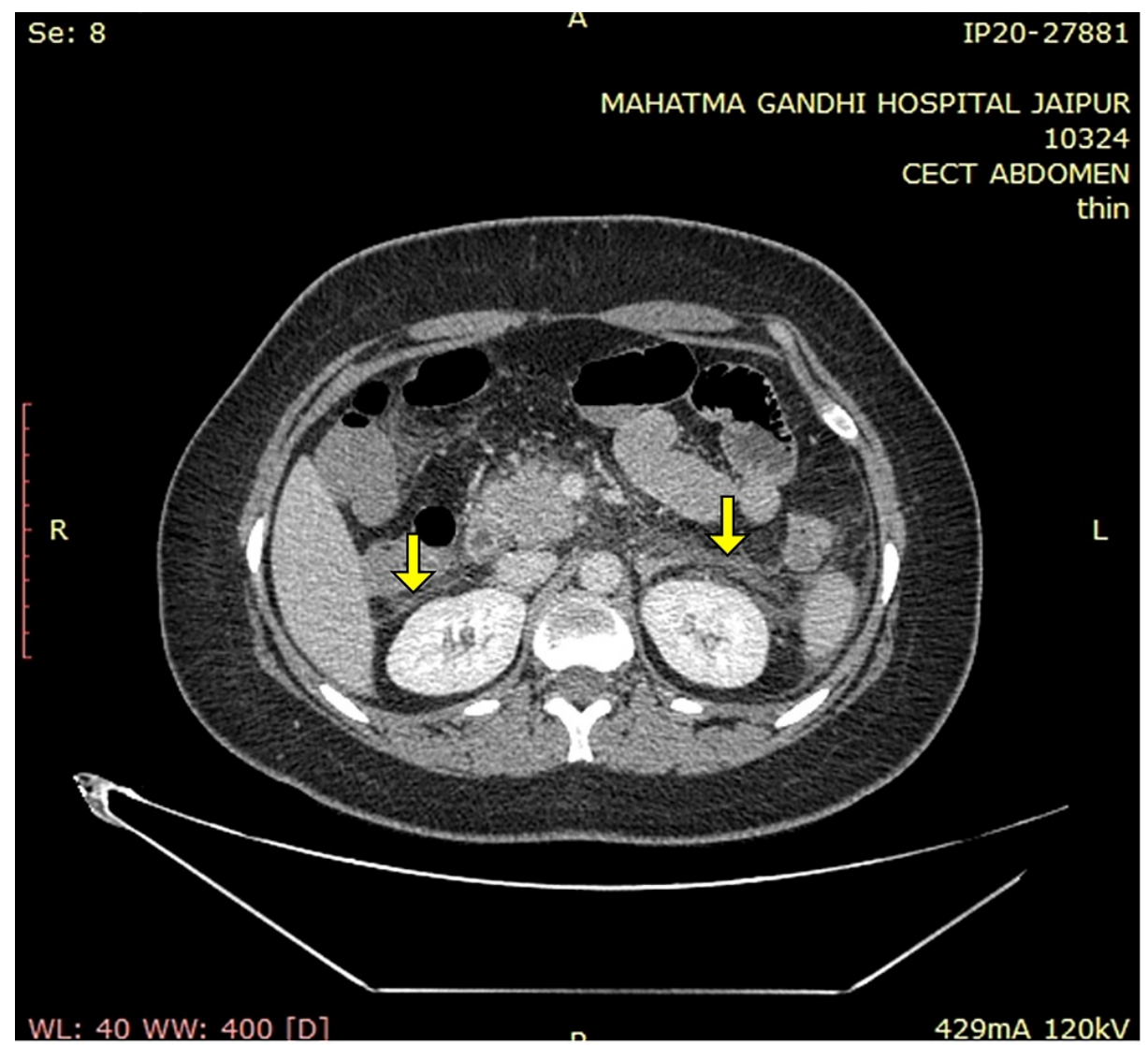

FIGURE 3: Contrast-enhanced CT of the abdomen (axial sections) showing bilateral renal and lateroconal fascial thickening (solid yellow arrows). 


\section{Cureus}

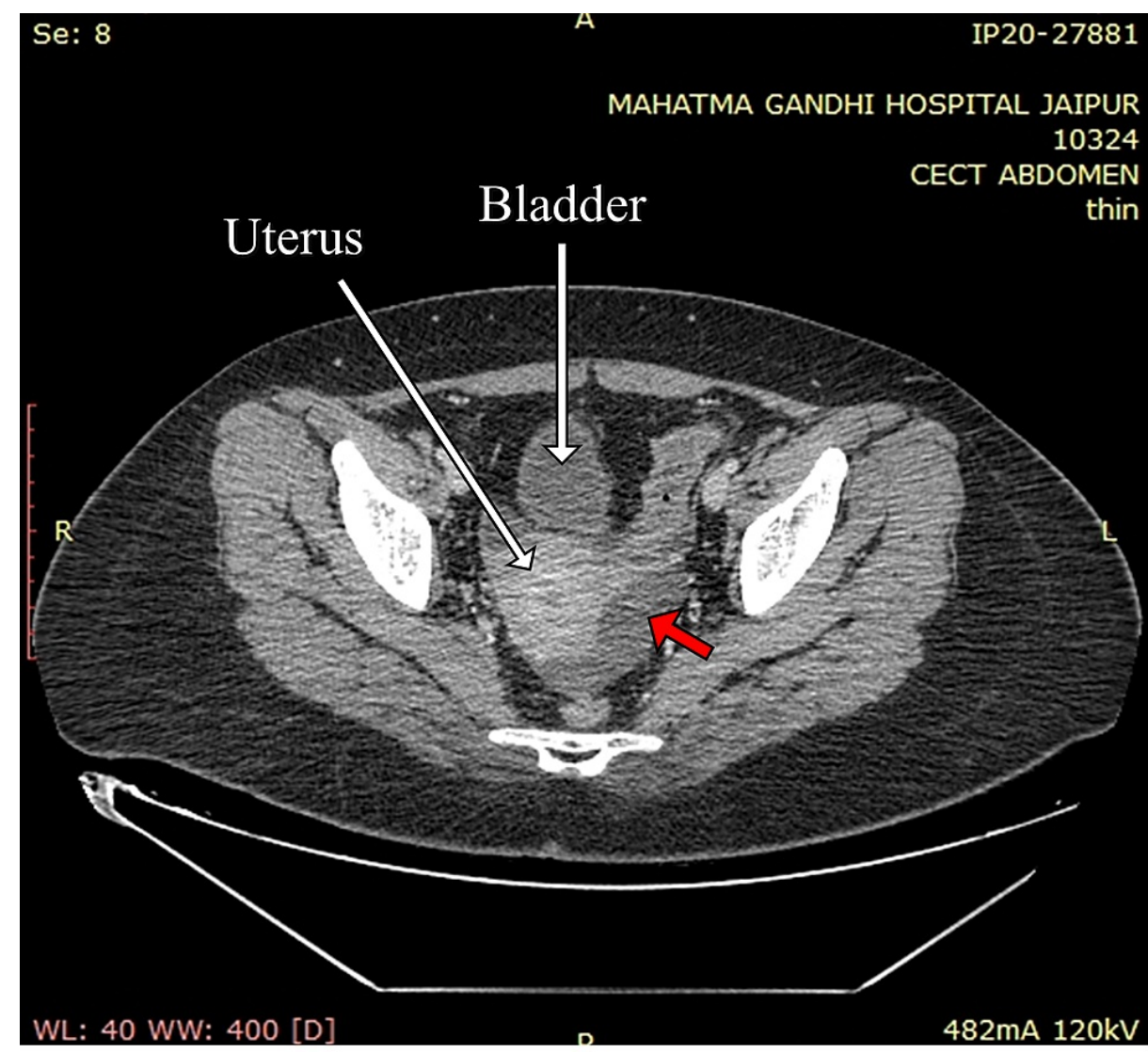

FIGURE 4: Contrast-enhanced CT of the abdomen (axial section) showing free fluid in the pelvis and ascites (solid red arrow).

The high-resolution CT of the chest showed patchy ground-glass opacities in the left lung, CO-RADS 6 ([COVID-19 Reporting and Data System] RT-PCR positive), CT severity score of 3/25 (Figures 5A, 5B), and bilateral pleural effusion (left > right) (Figure 6 ).

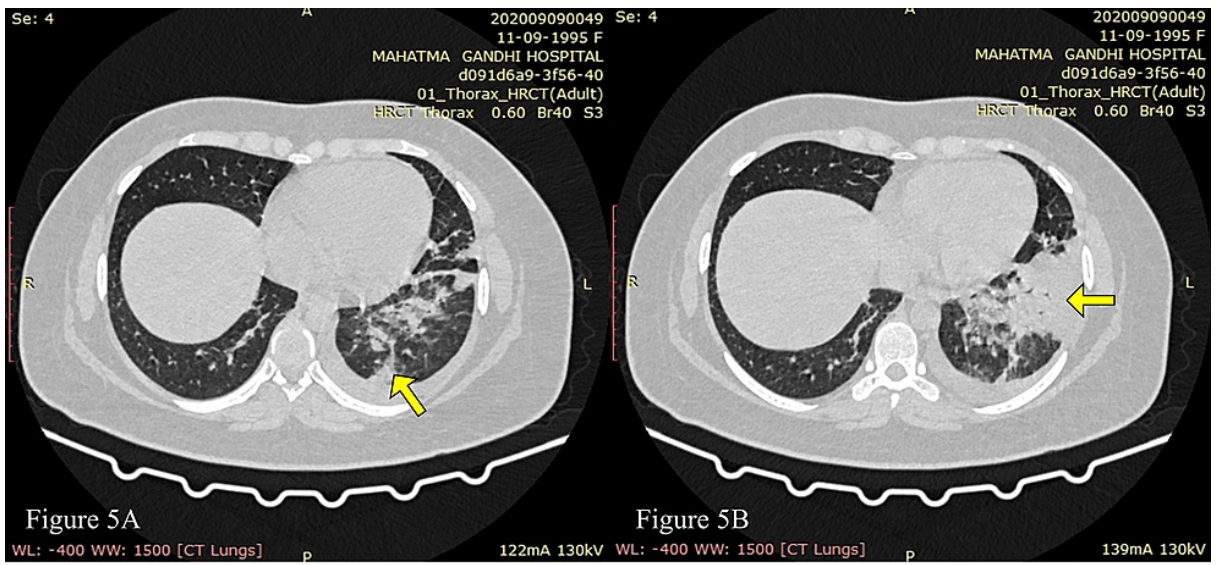

FIGURE 5: HRCT of the chest lung window (axial sections) showing ground-glass opacities and consolidation predominantly involving the left lower lobe of the lung (solid yellow arrows). CT severity score: $3 / 25$. HRCT, high-resolution CT 


\section{Cureus}

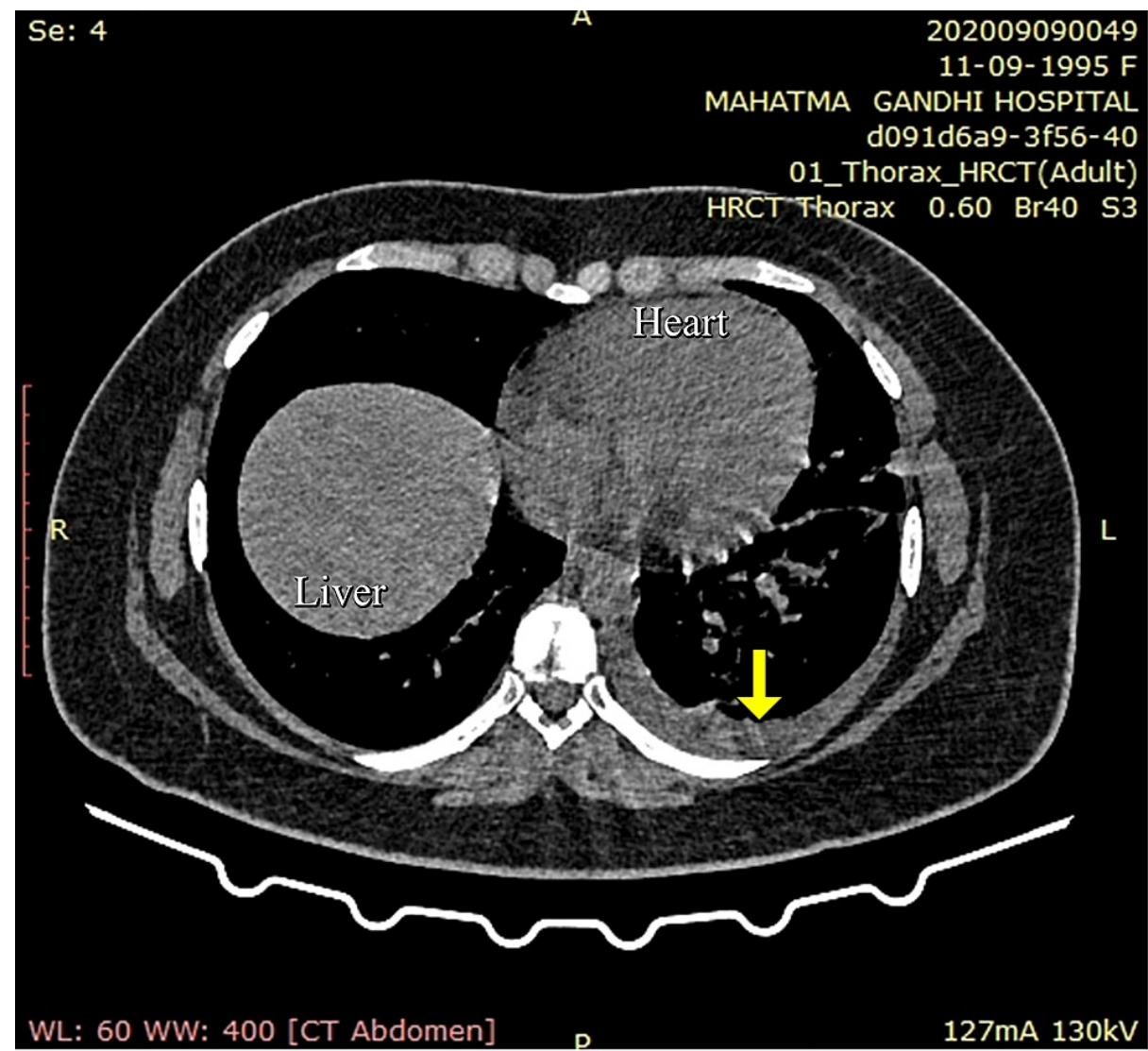

FIGURE 6: HRCT of the chest mediastinal window (axial section) showing left pleural effusion (solid yellow arrow).

HRCT, high-resolution CT

A screening 2D-ECHO (echocardiography) indicated no regional wall motion abnormality and a left ventricular ejection fraction of $60 \%$. She was managed conservatively and kept nil per os with nasogastric suction, judicious intravenous (IV) fluids, IV antibiotics (piperacillin-tazobactam and metronidazole), IV methylprednisolone $40 \mathrm{mg}$ once daily, subcutaneous enoxaparin $60 \mathrm{mg}$ twice daily, and other supportive measures. On her third day of admission (day 11 of illness), she required transfer to the intensive care unit for respiratory distress and increased oxygen requirement. She was also switched to IV imipenem and started on IV remdesivir. The possibility of microthrombi formation in the pulmonary circulation (highly elevated D-dimer: $11,100 \mathrm{ng} / \mathrm{mL}$ ), leading to pulmonary hypertension and myocardial stress (elevation of NT-proBNP [N-terminal-pro-B-type natriuretic peptide]: $3,990 \mathrm{pg} / \mathrm{mL}$ ) was kept, and the patient was started on IV furosemide $20 \mathrm{mg}$ once daily. The patient started showing significant improvement in abdominal pain and shortness of breath, and her oxygen requirement started decreasing. Her repeat COVID RT-PCR was negative on days 13 and 14 of illness (days 5 and 6 of admission), and she was able to tolerate oral liquid diet. She was off oxygen support and able to tolerate full oral diet by day 7 of admission and was discharged on day 9 of admission. A timeline of events (symptoms, treatment initiated) prior to admission is depicted (Figure 7). A graphical representation of her CRP (C-reactive protein), D-dimer, and NT-proBNP during the course of illness (after admission) is depicted (Figure 8).
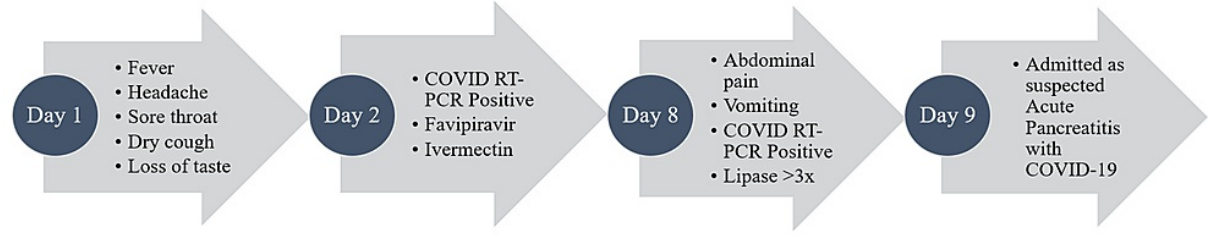

FIGURE 7: Timeline of symptoms: treatment initiated prior to admission. 


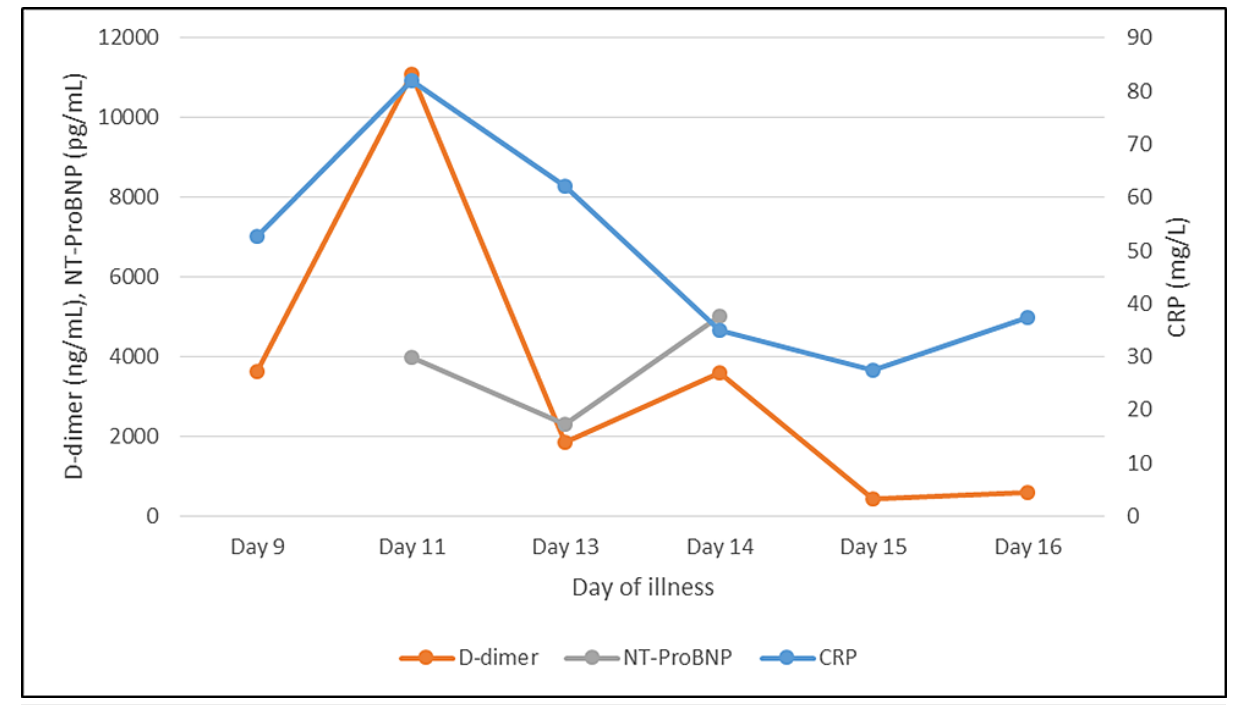

FIGURE 8: Graphical representation of CRP, D-dimer, and NT-proBNP values during the course of illness (after admission).

CRP, C-reactive protein; NT-proBNP, N-terminal-pro-B-type natriuretic peptide

\section{Discussion}

Patients with COVID-19 frequently present with gastrointestinal symptoms. In a report of over 3,70,000 cases of COVID-19, 19\% had diarrhea, $12 \%$ had nausea/vomiting, and 7.6\% had abdominal pain [7]. A metaanalysis and systematic review of 60 studies of gastrointestinal manifestations of severe acute respiratory syndrome coronavirus 2 (SARS-CoV-2) infection estimated the pooled prevalence of gastrointestinal symptoms at $17.6 \%$ overall (13\% for diarrhea, $10 \%$ for nausea/vomiting, and $9 \%$ for abdominal pain) [8], particularly higher in the severely ill. However, to date, there have not been many case reports of acute pancreatitis in COVID-19.

The diagnosis of acute pancreatitis requires two out of the following three features (as per the revised Atlanta classification) [9]: (1) abdominal pain consistent with acute pancreatitis, (2) serum lipase or amylase activity at least three times greater than the upper limit of normal, and (3) characteristic findings of acute pancreatitis on contrast-enhanced CT or MRI or USG. The common causes of pancreatitis, which were ruled out in this case, included biliary tract disease (e.g. gallstones), alcohol, hypertriglyceridemia, hypercalcemia, trauma, history of surgical procedures/post-ERCP (endoscopic retrograde cholangiopancreatography), developmental anomalies, and tumors. Drug-induced pancreatitis has not been reported with either ivermectin or favipiravir. Infections with viruses, helminths, and protozoa are known to cause pancreatitis, and viral infections such as those from hepatitis viruses, Coxsackie viruses, Echoviruses, mumps virus, cytomegalovirus, Epstein-Barr virus, Varicella Zoster virus, and influenza virus (H1N1) are the most common infectious cause (65\% cases) of acute pancreatitis [10].

Anand et al. [11] reported the development of acute pancreatitis in a patient 10 days after the first positive PCR report. The patient was readmitted after discharge with fever, abdominal pain, and constipation, and CT obtained on day 3 of readmission indicated acute pancreatitis [11]. They raised the possibility of an association between this novel coronavirus and acute pancreatitis [11]. The temporal presentation of acute pancreatitis in their case matches that of our case; however, a serum amylase level test was not done in their patient on admission.

Another case report of acalculous acute pancreatitis in a COVID-19 patient was reported by Meireles et al. [12], where the patient developed nausea, vomiting, and pain abdomen on the 11th day of the disease. Her investigations revealed a 10-fold rise in her amylase and lipase levels, and imaging did not reveal any evidence of calculi or ischemia [12]. They suggested that pancreatic involvement likely arose from an immune inflammatory response to the virus (rather than direct viral invasion) given the temporal association between the development of pancreatitis and the clinical picture [12].

Hadi et al. [13] described the cases of three family members admitted with COVID-19, two of whom developed severe acute pancreatitis. The authors did not find a reason as to why only two of the five cases in the family developed acute pancreatitis. Both of them had severe pancreatitis with acute respiratory distress syndrome (ARDS) and multi-organ failure, and whether severe pancreatitis caused the ARDS and multiorgan failure or COVID-19 caused it could not be ascertained [13]. Kumaran et al. [14] reported the case of a 67-year-old hypertensive female who presented with one-day history of epigastric pain, nausea, and 
vomiting and was found to have acute necrotising pancreatitis after due investigations. In that case also, common causes of acute pancreatitis were ruled out, but the patient did have a positive history of superior mesenteric artery stenosis one year ago, for which she underwent surgery and was on apixaban. She later developed tachypnea and hypoxia and was found to be COVID-19 positive [14]. It highlights the fact that patients with COVID-19 may even present with pancreatitis, and the worsening of respiratory system after the development of acute pancreatitis in their case matches that of our case.

The presence of viral RNA in fecal specimens and gastrointestinal epithelium despite negative respiratory tests has led to the suggestion of a possible feco-oral transmission route for SARS-CoV-2 [15]. The expression of ACE-2 ([angiotensin-converting enzyme 2] the host receptor for SARS-CoV-2 entry) is very high in the pancreas and gastrointestinal epithelium [16].

The endocrine part of the pancreas strongly expresses ACE-2, whereas exocrine tissues are only weakly positive; consequently, there are very few reports of pancreatitis in patients with SARS [17] or COVID-19. Wang et al. [18] reported that 17\% patients of COVID-19 had evidence of pancreatic injury (defined as elevation of pancreatic enzymes), and two-thirds of those patients had abnormal blood glucose levels [18]. They attributed this injury possibly due to either direct cytopathic effect of SARS-CoV-2 or mediated by the immune response directed against SARS-CoV-2 [18]. In another study by Liu et al. [19] of 121 patients with COVID-19, only one out of 54 mild cases had pancreatic enzyme elevation, but in severe cases, 12 out of 64 cases (17.9\%) had enzyme elevation, but no case had necrotising pancreatitis [19]. Clinicians should have a high index of suspicion for pancreatic injury in patients with COVID-19 (especially in those with pancreatic enzyme elevation), as it can be a life-threatening complication.

There are some lessons to be learned here from the 2002-2004 SARS epidemic. The pancreatic involvement in SARS was not only limited to pancreatic enzyme elevation. The development of acute diabetes in 20 out of 39 patients of SARS (who were initially non-diabetic) was noted by Yang et al. [17]. Of those 20 patients, 18 returned to normoglycemia in the three-year follow-up of the study [17]. This indicated that SARS-CoV-1 caused damage to pancreatic islets and caused acute diabetes. Considering the genetic similarities between SARS-CoV-1 and 2, the potential for permanent pancreatic damage, chronic pancreatitis, and acute or chronic diabetes due to SARS-CoV-2 cannot be overlooked.

Hanley et al. [20] reported the histopathological post-mortem findings in 10 patients over the age of 18 years who died due to COVID-19. The most consistent findings were diffuse alveolar damage, thrombosis, and immune cell depletion primarily affecting the lungs, hearts, and kidneys [20]. But the unexpected finding was pancreatitis in two patients, with the first having gross necrotising hemorrhagic pancreatitis and the second having only microscopic evidence of acute inflammation in the pancreas [20]. While it was not obvious what determines the spread of SARS-CoV-2 outside the respiratory tract (as the sites of highest ACE-2 expression did not correlate with severity of pathological involvement), it possibly depends on several other factors such as cellular receptor status, route of transmission, and host factors (genetic makeup, TMPRSS2 expression/polymorphism, etc.) [20].

\section{Conclusions}

Respiratory, cardiac, renal, and multi-organ failure, as well as thrombosis are the most common causes of mortality in patients with COVID-19. Acute pancreatitis can be a rare cause of mortality, especially in those with moderate-to-severe disease. Patients with COVID-19 may be screened for pancreatic injury, as it may have an effect on prognosis. Larger studies are required to confirm the cause and pathogenesis of acute pancreatitis in COVID-19.

\section{Additional Information}

\section{Disclosures}

Human subjects: Consent was obtained or waived by all participants in this study. Institutional Ethics Committee of Mahatma Gandhi Medical College and Hospital issued approval MGMCH/IEC/JPR/2021/271. Subject:- "-A case report on acute pancreatitis in a patient with coronavirus disease 2019 (COVID-19) pneumonia.” submitted for ethics committee approval. Dear Sir/Madam, Your letter for permission to conduct the above mentioned study was reviewed and discussed in the meeting of the Institutional Ethics Committee held on 6th March 2021. The following members of the ethics committee were present during the meeting: Dr. R.P. Kothari, Retd. Professor, General Medicine - CHAIRMAN * Dr. Rajendra kumar Sureka , PHOD, Neurology, MGMC\&H, Jaipur- MEMBER SECRETARY Dr. Puneet Rijhwani, PHOD, General Medicine, MGMC\&H, Jaipur - Clinician Dr. R.C. Gupta, Professor-Anaesthesia \& Additional Medical Superintendent, MGMC\&H, Jaipur - Clinician Dr. B.N. Sharma, PHOD, Community Medicine, MGMC\&H, Jaipur - Social Scientist Dr. Munish Kumar Kakkar, PHOD, Paediatrics, MGMC\&H, Jaipur - Clinician Dr. Anusha Vohara ,PHOD ,Pharmacology- Basic Medical Scientist Dr. Monica Jain Member-EC, Associate Professor, Department of Pharmacology, SMSMC, Jaipur (Basic Medical Scientist) Mr. Siddhant Jain , Advocate-Legal Advisor - Legal Expert Dr. Manju Bhaskar, Associate Professor, Psychiatry, MGMC\&H, Jaipur. - Clinician Dr. Amitabh Dube, Member Secretary-EC, Senior Professor, Department of Physiology, SMSMC, Jaipur. (Basic Scientist) Dr.(Mrs.)Lata Joshi, Lay Person from the Community-Lay Person. The Research Project in 
prescribed format A,B and C submitted by you were reviewed by the committee. The Committee approves the above mentioned study. The Committee fee of the IEC is exempted. This is certified that investigator or his/her representative was not present during the decision making procedure /discussion. The Ethics committee expects to be informed about: • The progress of the study time as well as about the completion of the study. • Any changes in the protocol and patient information/informed consent. • You will strictly follow the guidelines of schedule Y, ICMR, ICH-GCP, instructions of DCGI New Delhi, and also the SOP of this institution. - In case of any deviation, default or any unlawful activity, your study will be discontinued under intimation to the DCGI and ICMR New Delhi. Conflicts of interest: In compliance with the ICMJE uniform disclosure form, all authors declare the following: Payment/services info: All authors have declared that no financial support was received from any organization for the submitted work. Financial relationships: All authors have declared that they have no financial relationships at present or within the previous three years with any organizations that might have an interest in the submitted work. Other relationships: All authors have declared that there are no other relationships or activities that could appear to have influenced the submitted work.

\section{References}

1. WHO Director-General's remarks at the media briefing on 2019-nCoV on 11 February . (2020). Accessed: November 22, 2020: https://www.who.int/dg/speeches/detail/who-director-general-s-remarks-at-themedia-briefing-on-2019-ncov-on-11-februa....

2. WHO Director-General's opening remarks at the media briefing on COVID-19 - 11 March 2020 . (2020). Accessed: November 22, 2020: https://www.who.int/dg/speeches/detail/who-director-general-s-openingremarks-at-the-media-briefing-on-covid-19---11-....

3. COVID-19 Weekly Epidemiological Update - 5 January . (2021). Accessed: January 11, 2021: https://www.who.int/publications/m/item/weekly-epidemiological-update---5-january-2021

4. Oran DP, Topol EJ: Prevalence of asymptomatic SARS-CoV-2 infection: a narrative review . Ann Intern Med. 2020, 173:362-7. 10.7326/M20-3012

5. Wu Z, McGoogan JM: Characteristics of and important lessons from the coronavirus disease 2019 (COVID19) outbreak in China: summary of a report of 72314 cases from the Chinese Center for Disease Control and Prevention. JAMA. 2020, 323:1239-42. 10.1001/jama.2020.2648

6. Wise J: Covid-19: study reveals six clusters of symptoms that could be used as a clinical prediction tool . BMJ. 2020, 370:2911. 10.1136/bmj.m2911

7. Stokes EK, Zambrano LD, Anderson KN, et al.: Coronavirus disease 2019 case surveillance - United States, January 22-May 30, 2020. MMWR Morb Mortal Wkly Rep. 2020, 69:759-65. 10.15585/mmwr.mm6924e2

8. Cheung KS, Hung IFN, Chan PPY, et al.: Gastrointestinal manifestations of SARS-CoV-2 infection and virus load in fecal samples from a Hong Kong cohort: systematic review and meta-analysis. Gastroenterology. 2020, 159:81-95. 10.1053/j.gastro.2020.03.065

9. Banks PA, Bollen TL, Dervenis C, et al.: Classification of acute pancreatitis--2012: revision of the Atlanta classification and definitions by international consensus. Gut. 2013, 62:102-11. 10.1136/gutjnl-2012-302779

10. Imam Z, Simons-Linares CR, Chahal P: Infectious causes of acute pancreatitis: a systematic review . Pancreatology. 2020, 20:1312-2. 10.1016/j.pan.2020.08.018

11. Anand ER, Major C, Pickering O, Nelson M: Acute pancreatitis in a COVID-19 patient . Br J Surg. 2020, 107:e182. 10.1002/bjs.11657

12. Meireles PA, Bessa F, Gaspar P, Parreira I, Silva VD, Mota C, Alvoeiro L: Acalculous acute pancreatitis in a COVID-19 patient. Eur J Case Rep Intern Med. 2020, 7:001710. 10.12890/2020_001710

13. Hadi A, Werge M, Kristiansen KT, Pedersen UG, Karstensen JG, Novovic S, Gluud LL: Coronavirus disease-19 (COVID-19) associated with severe acute pancreatitis: case report on three family members. Pancreatology. 2020, 20:665-7.10.1016/j.pan.2020.04.021

14. Kumaran NK, Karmakar BK, Taylor OM: Coronavirus disease-19 (COVID-19) associated with acute necrotising pancreatitis (ANP). BMI Case Rep. 2020, 13:237903. 10.1136/bcr-2020-237903

15. Xiao F, Tang M, Zheng X, Liu Y, Li X, Shan H: Evidence for gastrointestinal infection of SARS-CoV-2. Gastroenterology. 2020, 158:1831-3. 10.1053/j.gastro.2020.02.055

16. Harmer D, Gilbert M, Borman R, Clark KL: Quantitative mRNA expression profiling of ACE 2, a novel homologue of angiotensin converting enzyme. FEBS Lett. 2002, 532:107-10. 10.1016/s0014-5793(02)03640 2

17. Yang JK, Lin SS, Ji XJ, Guo LM: Binding of SARS coronavirus to its receptor damages islets and causes acute diabetes. Acta Diabetol. 2010, 47:193-9. 10.1007/s00592-009-0109-4

18. Wang F, Wang H, Fan J, Zhang Y, Wang H, Zhao Q: Pancreatic injury patterns in patients with coronavirus disease 19 pneumonia. Gastroenterology. 2020, 159:367-70. 10.1053/j.gastro.2020.03.055

19. Liu F, Long X, Zhang B, Zhang W, Chen X, Zhang Z: ACE2 expression in pancreas may cause pancreatic damage after SARS-CoV-2 infection. Clin Gastroenterol Hepatol. 2020, 18:2128-30. 10.1016/j.cgh.2020.04.040

20. Hanley B, Naresh KN, Roufosse C, et al.: Histopathological findings and viral tropism in UK patients with severe fatal COVID-19: a post-mortem study. Lancet Microbe. 2020, 1:245-53. 10.1016/S2666-

5247(20)30115-4 